\title{
COMMISSION 37 STAR CLUSTERS AND ASSOCIATIONS
}

\author{
AMAS STELLAIRES ET ASSOCIATIONS
}

\author{
PRESIDENT \\ VICE-PRESIDENT \\ PAST PRESIDENT \\ MEMBERS
}

\author{
Ata Sarajedini \\ Rainer Spurzem \\ Georges Meylan \\ Russell D. Cannon, Vittorio Castellani, \\ Gary S. Da Costa, Kyle McC. Cudworth \\ Licai Deng, Miroslav Giersz, \\ Despina Hatzidimitriou, Charles J. Lada
}

\section{PROCEEDINGS BUSINESS MEETING on 17 August 2006}

\section{Summary}

The Commission business meeting was held on 17 August 2006. Approximately 20 people attended, including the Commission vice-president, Rainer Spurzem. The sole member of the Commission Organizing Committee that was present was Gary Da Costa, who, along with Patricia Whitelock, the outgoing president of Division VII and Commission 33, served as chairs of the meeting.

The nominations of Despina Hatzidimitriou as Commission president and Charles Lada as vice-president were approved by the members of the Commission Organizing Committee prior to the business meeting. Those present at the business meeting agreed with this recommendation.

The membership of the Organizing Committee (OC) of Commission 37 was also revised. Rainer Spurzem and Mirek Giersz requested to leave the OC; they were replaced by YoungWook Lee and Monica Tosi. Vittorio Castellani passed away earlier in the year. Thus, the OC is now composed of Russell Cannon, Kyle Cudworth, Gary Da Costa, Licai Deng, Young-Wook Lee, Ata Sarajedini (past president), and Monica Tosi. 